\title{
Studies on Chitin. V. Kinetics of Deacetylation Reaction
}

\author{
Takanori Sannan, Keisuke Kurita, and Yoshio Iwakura \\ Department of Industrial Chemistry, Faculty of Engineering, \\ Seikei University, Musashino, Tokyo 180, Japan.
}

(Received June 28, 1977)

\begin{abstract}
KEY WORDS Chitin / Poly $(N$-acetyl-D-glucosamine) / Polysaccharide / Hydrolysis / Kinetics / Rate Constant / Activation Energy / Deacetylation / Acetamide Group / Amino Group /
\end{abstract}

Chitin, poly( $N$-acetyl-D-glucosamine), is a naturally abundant polysaccharide and is one of the most important materials in Nature, but relatively little attention has been paid to its reactions and utilization probably because of its intractability. Although both acidic and alkaline hydrolyses of chitin are widely used for obtaining chitin oligosaccharides and chitosan, respectively, only the acidic hydrolysis has been kinetically studied by Meyer, et al., to determine the activation energy for hydrolytic cleavage of the glycosidic linkage. ${ }^{1}$

The modification of chitin by alkaline hydrolysis, leading to possible utilization, has been of interest to us for some time and we have already reported about its solubility change through alkaline hydrolysis. ${ }^{2-4}$ In the course of our study, we found it of interest to study the kinetics of the alkaline hydrolysis of pendant acetamide groups attached to the polysaccharide backbone.

The work described in this paper is part of the investigation concerning the reactions of chitin and it deals with the kinetic study of a homogeneous alkaline hydrolysis of chitin directed toward the hydrolysis of the acetamide group.

\section{EXPERIMENTAL}

Chitin was isolated from the shells of Penaeus japonicus by Hackman's method. ${ }^{5}$ An alkali chitin solution was prepared with a polymer concentration of $1 \%$ and an $\mathrm{NaOH}$ concentration of $10 \%$.

Deacetylation of chitin was achieved by standing the sololution at constant temperature and this was followed by titrating the $10-\mathrm{g}$ aliquot of the solution with $0.1-N \mathrm{HCl}^{3}$.

\section{RESULTS AND DISCUSSION}

From our hydrolytic study of chitin we found that in order to keep the alkali chitin in homogeneous solution, the alkali concentration was required to be more than $8 \%,{ }^{2}$ and that up to $40^{\circ} \mathrm{C}$ the deacetylation could be achieved homogeneously." Thus, the alkaline hydrolysis was carried out at 25,30 , and $40^{\circ} \mathrm{C}$ with the alkali concentration being $10 \%$. The extent of deacetylation was determined by successive titration of the solutions with diluted hydrochloric acid. Table I tabulates the volumes of $0.1-N$

Table I. Titration of $10-\mathrm{g}$ aliquot of the alkali chitin solution with $0.1-N \mathrm{HCl}$

\begin{tabular}{|c|c|c|c|c|c|}
\hline \multirow{2}{*}{\multicolumn{2}{|c|}{ at $25^{\circ} \mathrm{C}$}} & \multicolumn{2}{|c|}{ Hydrolysis } & \multirow{2}{*}{\multicolumn{2}{|c|}{ at $40^{\circ} \mathrm{C}$}} \\
\hline & & & $30^{\circ} \mathrm{C}$ & & \\
\hline$\underset{\mathrm{hr}}{\text { Time }}$ & $\begin{array}{c}\text { Con- } \\
\text { sumed } \\
\text { volume, } \\
\mathrm{m} l\end{array}$ & $\underset{\mathrm{hr}}{\text { Time, }}$ & $\begin{array}{c}\text { Con- } \\
\text { sumed } \\
\text { volume, } \\
\mathrm{m} l\end{array}$ & $\underset{\text { hr }}{\text { Time, }}$ & $\begin{array}{c}\text { Con- } \\
\text { sumed } \\
\text { volume, } \\
\mathrm{m} l\end{array}$ \\
\hline 24 & 2.1 & 22 & 2.3 & 15 & 2.8 \\
\hline 48 & 2.5 & 43 & 3.0 & 19 & 3.1 \\
\hline 72 & 2.9 & 67 & 3.7 & 27 & 3.6 \\
\hline 95 & 3.3 & 91 & 4.0 & 39 & 4.1 \\
\hline 165 & 3.8 & 115 & 4.2 & & \\
\hline 580 & 4.6 & & & & \\
\hline
\end{tabular}


hydrochloric acid consumed in the acidimetry, corresponding to the amounts of the amino group formed by the alkaline hydrolysis of the acetamide group.

The kinetic order of the alkaline deacetylation of chitin may be second order with respect to the acetamide group and sodium hydroxide. However, since the latter is present in excess in this case, the rate of the reaction is assumed to be proportional to the concentration of the acetamide group, i.e., a pseudo-first-order reaction. The rate constant $k$ may therefore be expressed as follows

$$
k=\frac{2.303}{t} \log \frac{a}{a-x}
$$

where $a$ is the original concentration of the acetamide group and $x$ is the concentration of the resulting amino group at any time $t$.

In order to obtain the rate constant $k$ directly from the titration volumes given in Table I, eq 1 is rewritten as

$$
k=\frac{2.303}{t-t_{0}} \log \frac{V_{\infty}-V_{0}}{V_{\infty}-V}
$$

where $V_{0}$ is the volume $(\mathrm{m} l)$ of $0.1-N$ hydrochloric acid consumed by a 10 -g aliquot of the alkali chitin solution at the first time $t_{0}, V$ is the volume at time $t$, and $V_{\infty}$ is the volume after prolonged hydrolysis, which was found to be $4.6 \mathrm{ml}$ after $580 \mathrm{hr}$ at $25^{\circ} \mathrm{C}$. This substitution is based on the view that $\left(V_{\infty}-V_{0}\right)$ and $\left(V_{\infty}-V\right)$ are proportional to $(a)$ and $(a-x)$, respectively.

The values of $\left(V_{\infty}-V\right)$ were plotted against time on a semilogarithmic graph paper at each temperature. The plots gave straight lines as

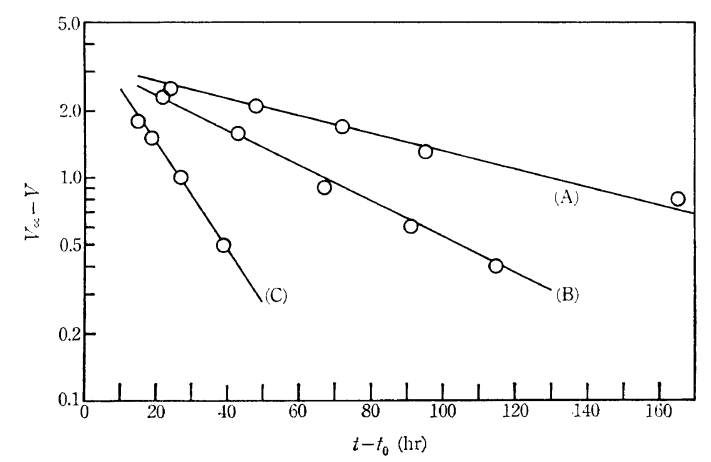

Figure 1. The plots of $\log \left(V_{\infty}-V\right) v s$. $\left(t-t_{0}\right)$; hydrolyzed at $25^{\circ} \mathrm{C}(\mathrm{A}), 30^{\circ} \mathrm{C}(\mathrm{B})$, and $40^{\circ} \mathrm{C}(\mathrm{C})$.

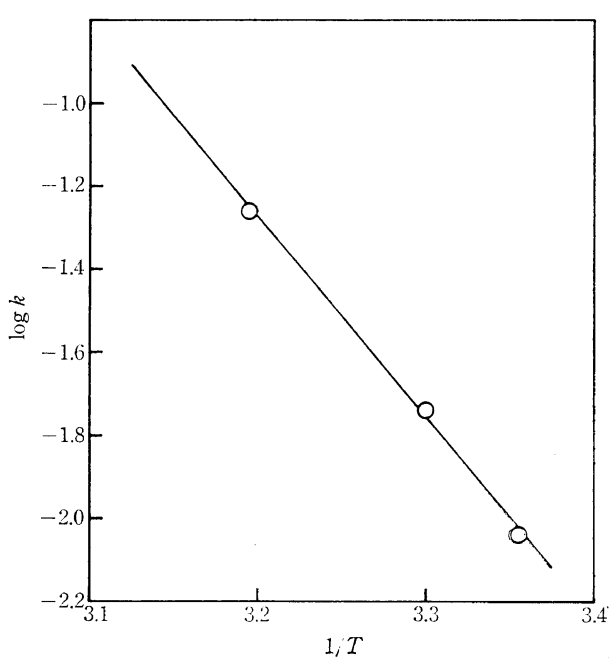

Figure 2. The Arrhenius plot of $\log k v$ s. $1 / T$.

shown in Figure 1. These linear relationships between $\log \left(V_{\infty}-V\right)$ and time appeared to support the speculation that the deacetylation of chitin in the homogeneous alkali chitin solution would be a pseudo-first-order reaction. The values of the rate constants were calculated by multiplying the slopes of these linear rate plots by 2.303 , and were found to be $9.2 \times 10^{-3} \mathrm{hr}^{-1}$ at $25^{\circ} \mathrm{C}, 1.8 \times 10^{-2} \mathrm{hr}^{-1}$ at $30^{\circ} \mathrm{C}$, and $5.5 \times 10^{-2}$ $\mathrm{hr}^{-1}$ at $40^{\circ} \mathrm{C}$.

The activation energy for the deacetylation of chitin was then evaluated from 4.574 time the slope of the straight line of the Arrhenius plot $(\log k-1 / T)$ shown in Figure 2. It was found to be $22 \mathrm{kcal} / \mathrm{mol}$.

It was considered worth while to compare the homogeneous alkaline hydrolysis behaviors of poly( $N$-acetyl-D-glucosamine) with that of its monomer, $N$-acetyl-D-glucosamine. The deacetylation of $N$-acetyl-D-glucosamine was then carried out under the same conditions. During the hydrolysis, however, the solution took on a brown color and, furthermore, became a deeper brown at higher temperatures. Although the aliquot was similarly titrated, it was found difficult to obtain reliable titration curves. Thus comparison of the reactivity with that of the polysaccharide was not possible. The deep coloration probably resulted from some decomposition of the resulting D-glucosamine, since it is reported to be unstable in solution. ${ }^{5}$ 
Studies on Chitin. V. Kinetics of Deacetylation Reaction

\section{REFERENCES}

1. K. H. Meyer and H. Wehrli, Helv. Chim. Acta, 20, 353 (1937).

2. T. Sannan, K. Kurita, and Y. Iwakura, Makromol. Chem., 176, 1191 (1975).
3. T. Sannan, K. Kurita, and Y. Iwakura, Macromol. Chem., 177, 3589 (1976).

4. K. Kurita, T. Sannan, and Y. Iwakura, Macromol. Chem., in press.

5. R. H. Hackman, Aust. J. Biol. Sci., 7, 168 (1954). 